\title{
Sound Propagation in Rigid Porous Media: Non-local Macroscopic Effects Versus Pores Scale Regime
}

\author{
Claude Boutin
}

\begin{abstract}
This paper investigates how the regime (quasi-static, transient, out of equilibrium) of the phenomena occurring at pores scale determine the nature of the (non)-local effect-in time and/or space-involved in the macroscopic behavior of a porous medium. The study focuses on sound propagation examining - through the homogenization method of periodic media - situations of single porosity, Rayleigh scattering and double porosity. Nonlocality effects reveals the loss of a perfect quasi-static equilibrium free of volume loading at the local scale. The non-locality in time is due to phenomena in transient regime at the ERV scale, while non-locality in space is due to the non-homogeneity in space of the macrofields. The generality of the arguments lead to infer that the conclusions about non-locality versus pores scale regime, could be extended to other physical phenomena in heterogeneous media.
\end{abstract}

Keywords Acoustics - Porous media - Scattering - Double porosity - Higher gradient materials $\cdot$ Meta-materials $\cdot$ Non-local effects $\cdot$ Memory effects

\section{Introduction}

The sound propagation in air saturated rigid porous media (e.g., noise-absorbing materials) is usually described by the equivalent fluid model (Zwikker and Kosten 1949; Allard 1993) that captures the physical features of the acoustic wave ( $P_{2}$ wave in the sense of Biot (1956)). This modeling efficiently describes acoustic waves, provided that the wavelength is significantly larger than the characteristic size of the elementary representative volume (ERV) of the media, and that the microstructure may be characterized by a "single" pores size. The parameters of the equivalent fluid model (dynamic permeability, effective compressibility) are frequency dependent and therefore memory effects arise. Hence, the macro behavior is "non-local in time" in the sense that the mass flux does not depend only on the instantaneous

C. Boutin (ख)

Université de Lyon, DGCB CNRS 3237, Ecole Nationale des Travaux Publics de 1'Etat, 69518 Vaulx-en-Velin Cedex, France

e-mail: claude.boutin @entpe.fr 
pressure gradient in the ERV—as in the stationary Darcy's law—but also involves the history of the pressure gradient in the ERV.

When the wavelength is not too large compared to the ERV size (Rayleigh scattering), or when the ERV presents a hierarchical microstructure (double porosity) the usual modeling has to be revisited (Auriault and Boutin 1994; Boutin et al. 1998; Boutin and Auriault 1993; Boutin 2007). These situations introduce new phenomena at the ERV scale and the corresponding macroscopic description is enriched by either time or space non-local effects. The denomination "non-locality in space" means that the mass flux does not depend only on the pressure gradient in the ERV—as in the classical Darcy's law-but involves also the successive pressure gradient, or equivalently the pressure gradient in the neighbor ERVs of the considered ERV. Those deviations from the equivalent fluid model are supported by experiments, for instance Leclaire et al. (1996) for the scattering, and Only and Boutin (2003) for double porosity.

In practice, non-local effects closely interacts with the other phenomena and cannot be measured independently from laboratory or field experiments. Hence, the interest of understanding their origin is to provide physical arguments (and modeling) enabling to discriminate the possible nature of the deviation from the equivalent fluid model.

The aim of this paper is to investigate how the (non)-locality of the macroscopic description is related to the nature of equilibrium at the ERV scale. The homogenization method of periodic media (Sanchez Palencia 1980; Auriault et al. 2009), is well adapted to this study since this multi-scale asymptotic approach enables to build the macroscopic description from the knowledge of the physics at the local level (provided that a sufficiently good scale separation between macro and micro scales is fulfilled). This paper focuses on the specific case of sound propagation in rigid porous media. However, the generality and the convergence of the arguments derived from the analysis of single porosity media, Rayleigh scattering and double porosity media, lead to infer that the conclusions about non-locality versus local regime, could be extended to other physical phenomena in heterogeneous media.

The paper is organized as follows. The second part recalls the basic physics of gas and the principle of the homogenization method. The third and fourth parts, respectively, focus on long wave propagation and Rayleigh scattering in single porosity media, while the fifth part is devoted to long wave propagation in double porosity media. In these three cases, the conditions of emergence and the nature of non-local effects are discussed. A synthesis is drawn in conclusion.

\section{Features of Waves in Gas: Homogenization Method}

\subsection{Compression, Shear, and Thermal Waves in gas}

Let us recall the governing equations of a gas submitted to small harmonic perturbations (of frequency $f=\omega / 2 \pi$ ) from its equilibrium state (where the pressure, temperature, and density take the values $P^{\mathrm{e}}, T^{\mathrm{e}}$, and $\rho^{\mathrm{e}}$ ). The variables describing the perturbations are the variations of pressure, $p$, temperature, $\theta$, density, $\rho$, and the gas velocity $v,(D(v)$ is the strain rate). The parameters of the gas (see Table 1 for physical properties of air) are its viscosity, $\mu$, thermal conductivity, $\kappa$, specific heat ratio, $\gamma$, and mass capacity $c_{p}$. Considering a perfect gas, one has the relation $c_{p}\left(1-\frac{1}{\gamma}\right)=\frac{P^{\mathrm{e}}}{T^{\mathrm{e}} \rho^{\mathrm{e}}}$. Under perturbations of weak amplitude the balance equations can be linearized and take the form below (here and in the following 
Table 1 Physical parameters of air in ambient conditions

\begin{tabular}{llllllll}
\hline$P^{e}(\mathrm{~Pa})$ & $T^{e}(\mathrm{~K})$ & $\rho^{e}\left(\mathrm{~kg} / \mathrm{m}^{3}\right)$ & $C_{a}(\mathrm{~m} / \mathrm{s})$ & $\mu(\mathrm{Pa} \mathrm{s})$ & $\kappa(\mathrm{WKm})$ & $\rho^{e} c_{p}\left(\mathrm{~J} / \mathrm{Km}^{3}\right)$ & $\gamma$ \\
\hline $10^{5}$ & 293 & 1.2 & 343 & $1.810^{-5}$ & 0.026 & 1230 & 1.4 \\
\hline
\end{tabular}

the term $\exp (i \omega t)$ is omitted; $\nabla$ stands for the gradient, $\Delta$ for the Laplacian, dot stands for contraction, double dots for double contraction, etc.):

- Mass balance:

$$
\operatorname{div}(v)+i \omega \frac{\rho}{\rho^{\mathrm{e}}}=0
$$

- Momentum balance (Navier-Stokes equation):

$$
\operatorname{div}(2 \mu D(v))-\nabla p-i \omega \rho^{\mathrm{e}} v=0
$$

- Fourier equation (energy balance):

$$
\operatorname{div}(\kappa \nabla \theta)-i \omega\left(\rho^{\mathrm{e}} c_{p} \theta-p\right)=0
$$

- State equation of the gas

$$
\frac{p}{P^{\mathrm{e}}}=\frac{\rho}{\rho^{\mathrm{e}}}+\frac{\theta}{T^{\mathrm{e}}}
$$

Noting that the gas state equation enables the elimination of $\rho$ and that $\operatorname{div}(2 \mu D(v))=$ $\mu[\Delta(v)+\nabla \operatorname{div}(v)]$, the gas is driven by the three-coupled differential operators acting on the variables $p, v$, and $\theta$ :

$$
\begin{gathered}
\operatorname{div}(v)+i \omega\left(\frac{p}{P^{\mathrm{e}}}-\frac{\theta}{T^{\mathrm{e}}}\right)=0 \\
-\nabla p-i \rho^{\mathrm{e}} \omega v+\mu\left[\triangle(v)-i \omega \nabla\left(\frac{p}{P^{\mathrm{e}}}-\frac{\theta}{T^{\mathrm{e}}}\right)\right]=0 \\
i \omega\left(p-\rho^{\mathrm{e}} c_{p} \theta\right)+\operatorname{div}(\kappa \nabla \theta)=0
\end{gathered}
$$

The kernel of this set is constituted by three "pure" (or uncoupled) waves modes, each of them being associated to a characteristic length:

- The classical acoustic mode ( $P$-mode) of wave celerity $C_{a}=\sqrt{\frac{\gamma P^{\mathrm{e}}}{\rho^{\mathrm{e}}}}$. It corresponds to pure compression associated to pressure, isotropic deformation in the absence of viscosity and adiabatic regime. The acoustic wavelength $\lambda_{p}$, is derived from (5) and (6), taking $D(v)=\operatorname{div}(v) \mathrm{I}, \mu=0$ and assuming zero thermal flux, (i.e., $\kappa \nabla \theta=0$, thus $\rho^{\mathrm{e}} c_{p} \theta=p$ and $\left.\frac{p}{P^{\mathrm{e}}}-\frac{\theta}{T^{\mathrm{e}}}=\frac{p}{\gamma P^{\mathrm{e}}}\right)$ :

$$
\frac{\lambda_{p}}{2 \pi}=\sqrt{\frac{\gamma P^{\mathrm{e}}}{\rho^{\mathrm{e}}}} \frac{1}{\omega}=\frac{C_{a}}{\omega}
$$

- The diffusive mode of viscous deviatoric stress ( $S$-mode). The viscous layer thickness $\delta_{v}$, is derived from (5) in the absence of volume variation $(\operatorname{div}(v)=0)$ and assuming adiabatic regime:

$$
\delta_{v}=\left|\sqrt{\frac{\mu}{i \omega \rho^{\mathrm{e}}}}\right|
$$


- The diffusive mode of temperature ( $T$-mode). The thermal layer thickness $\delta_{t}$, is derived from (6) in the absence of pressure:

$$
\delta_{t}=\left|\sqrt{\frac{\kappa}{i \omega \rho^{\mathrm{e}} c_{p}}}\right|
$$

In gas, the viscous and thermal layer thicknesses, both related to Brownian motions, are of the same order $\left(\delta_{v} / \delta_{t} \approx 0.7\right.$ for air). Conversely, in ambient conditions and for usual frequency range (say less than $100 \mathrm{MHz}$ ), the acoustic wavelength is much larger than both viscous and thermal layers:

$$
2 \pi \delta_{v} / \lambda_{p}=\sqrt{\frac{\omega \mu}{\gamma P^{\mathrm{e}}}} \approx 10^{-5} \sqrt{f} \ll 1
$$

Consequently, in a domain of characteristic size $\ell$ such that:

$$
\lambda_{p} \gg \ell \leq \delta_{v} \approx \delta_{t}
$$

the physics is governed by a dynamic regime of the $S$ - and $T$ - modes and a quasi-static regime for the $P$-mode. This is precisely the situation encountered in classical poro-acoustics in medium frequency range as detailed in Sect. 3 .

\subsection{Basics of Homogenization Method}

The macroscopic representation of heterogeneous media makes sense only if there is a scale separation. This implies (Auriault 1991):

- that the material is regular enough to show an ERV. This is expressed by considering that the material is composed of repeated identical cells $\Omega$ of characteristic size $l$.

- that the phenomenon must vary according to a size $L$ larger than $l$. In acoustics, $L$ is related to the wavelength by $L=\lambda / 2 \pi$ (Boutin and Auriault 1990).

To capture the variations at the well-distinct lengths $L$ and $l$, two space variables are introduced (Benssoussan et al. 1978; Sanchez Palencia 1980): $x$ for the macro variations, $y$ for the micro variations, $x$ and $y$ being related by the scale ratio $\epsilon=l / L<<1 ; y=\epsilon^{-1} x$. It is worth mentioning that for a given medium, the actual physical scale ratio $\epsilon$ varies according to the wavelength, and therefore to the frequency.

The small parameter $\epsilon$ suggests seeking for the variables in the form of asymptotic expansions in powers of $\epsilon$ (where ${ }^{i} p(x, y)$ is the the $i$ th term of the expansion of $p(x, y)$, etc.,...):

$$
p(x, y)=\sum_{0}^{\infty} \epsilon^{i}{ }^{i} p(x, y) ; \quad \theta(x, y)=\sum_{0}^{\infty} \epsilon^{i} \theta(x, y) ; \quad v(x, y)=\sum_{0}^{\infty} \epsilon^{i}{ }^{i} v(x, y)
$$

The scale separation and material periodicity induces the same periodicity for the variables, thus the terms ${ }^{i} p,{ }^{i} \theta$ and ${ }^{i} v$ are $\Omega$-periodic according to $y$. Further, using the two space variables $x$ and $y=\epsilon^{-1} x$, the gradient $\nabla$ is changed into $\epsilon^{-1} \nabla_{y}+\nabla_{x}$, the Laplacian $\triangle$ becomes $\epsilon^{-2} \triangle_{y}+2 \epsilon^{-1} \triangle_{y x}+\triangle_{x}$, with $\triangle_{y x}=\partial_{x_{i} y_{i}}^{2}$, etc... The homogenization proceeds in three steps:

- first, perform a physical analysis and rescal the equations, using powers of $\epsilon$ for expressing the order of magnitude of the dimensionless terms,

- second, introduce the two-scale expansions in the rescaled two-scale equations and identify the terms of same power in $\epsilon$,

- third, solve the problems obtained in series, and derive the macro description from the global balance(s) of the cell. 


\section{Classical Poro-acoustics}

Consider now the gas within a rigid periodic single porosity medium (Fig. 1) of porosity $\phi=\frac{\Omega_{\mathrm{f}}}{\Omega}$ (where $\Omega_{\mathrm{f}}$ is the pore volume in the periodic cell of volume $\Omega$ ) and characteristic pores size $\ell$. Keeping the same notations, the governing equations of the small harmonic perturbations of the gas in the pores $\Omega_{f}$ are still given by (5-6-7). Now, this set has to be completed by the $\Omega$-periodicity of $v, p$, and $\theta$, and the boundary conditions on the gas-solid interface $\Gamma$, namely (here and in the following the notation $/ \Gamma$ means the value on $\Gamma$ ):

Adherence condition of the gas:

$$
v_{/ \Gamma}=0
$$

Isothermal condition imposed by the weak thermal impedance of air, $\kappa \rho^{\mathrm{e}} c_{p}$, compared to that of the solid:

$$
\theta_{/ \Gamma}=0
$$

\subsection{Harmonic Gas Motion in Pores: Rescaled Formulation}

The conditions on the pores boundary $\Gamma$ impose that both velocity and temperature vary at the pore scale. Consequently, physical variable varying macroscopically is the pressure. This imposes that the wavelength $\lambda_{p}$ related to the $P$-mode is much larger than the pores size. Hence, at pores scale the $P$-mode is in quasi-static regime. Denoting by $\Lambda$ the macroscopic wavelength, the scale separation imposes:

$$
O(L)=O(\Lambda /(2 \pi)) \gg \ell \quad \text { and } \quad \epsilon=O(2 \pi \ell / \Lambda)
$$

The richest physical case occurs when the $S$ - and $T$-modes are in transient regime at pore scale, i.e., equivalently, when $\ell$ is of the same order as the viscous and thermal layers: $\delta_{v} \approx \delta_{t}=O(\ell)=O(\epsilon L)$. To account for this situation, and taking the macroscopic wavelength as reference length, both viscous term in Navier-Stokes equation, and conduction term in Fourier equation have to be rescaled by $\epsilon^{2}$, while the mass balance (1) is unchanged

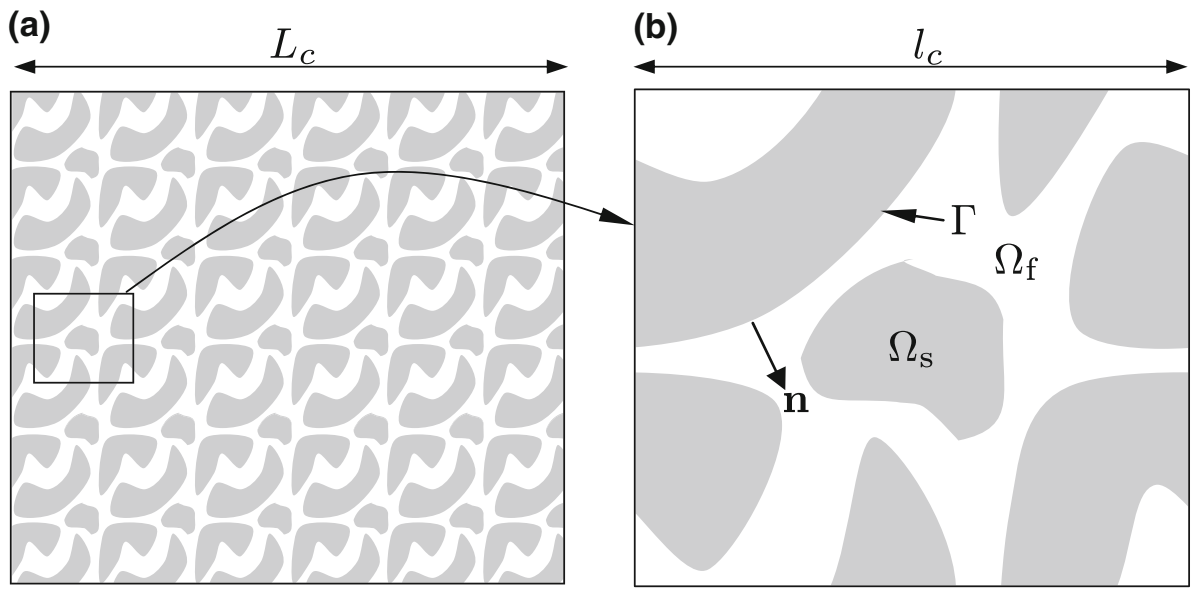

Fig. 1 Microstructure of a periodic single porosity media. a Porous media. b Periodic cell $\Omega$ of porous media. $\Omega_{\mathrm{f}}$ is the pore volume filled of gas. $\Gamma$ is the air-solid interface. $\phi=\Omega_{\mathrm{f}} / \Omega$ is the porosity 
(see for instance Boutin et al. (1998)). Thus, the gas is driven by the differential rescaled system:

$$
\begin{gathered}
G(p, v, \theta)=0 ; \quad G(p, v, \theta)=\operatorname{div}(v)+i \omega\left(\frac{p}{P^{\mathrm{e}}}-\frac{\theta}{T^{\mathrm{e}}}\right) \\
N(p, v, \theta)=0 ; \quad N(p, v, \theta)=-\nabla p-i \rho^{\mathrm{e}} \omega v+\epsilon^{2} \mu\left[\triangle(v)-i \omega \nabla\left(\frac{p}{P^{\mathrm{e}}}-\frac{\theta}{T^{\mathrm{e}}}\right)\right] \\
F(p, \theta)=0 ; \quad F(p, \theta)=i \omega\left(p-\rho^{\mathrm{e}} c_{p} \theta\right)+\epsilon^{2} \operatorname{div}(\kappa \nabla \theta)
\end{gathered}
$$

Then, the operators $G, N, F$ expressed with the two space variables are changed into $G_{x y}$, $N_{x y}, F_{x y}$, whose expansions are given by Eqs. (14) through (16):

$$
\begin{aligned}
G_{x y}(p, v, \theta)= & \epsilon^{-1} G^{-1}(v)+G^{0}(p, v, \theta) \\
& G^{-1}(v)=\operatorname{div}_{y}(v) \\
& G^{0}(p, v, \theta)=\operatorname{div}_{x}(v)+i \omega\left(\frac{p}{P^{\mathrm{e}}}-\frac{\theta}{T^{\mathrm{e}}}\right) \\
N_{x y}(p, v, \theta)= & \epsilon^{-1} N^{-1}(p)+N^{0}(p, v)+\epsilon N^{1}(p, v, \theta)+\epsilon^{2} N^{2}(p, v, \theta) \\
& N^{-1}(p)=-\nabla_{y} p \\
& N^{0}(p, v)=-\nabla_{x} p-i \omega \frac{\rho^{\mathrm{e}}}{\mu} \mu v+\triangle_{y}(\mu v) \\
& N^{1}(p, v, \theta)=2 \triangle_{y x}(\mu v)-i \omega \frac{\mu}{P^{\mathrm{e}}} \nabla_{y}\left(p-\frac{P^{\mathrm{e}}}{T^{\mathrm{e}}} \theta\right) \\
& N^{2}(p, v, \theta)=\triangle_{x}(\mu v)-i \omega \frac{\mu}{P^{\mathrm{e}}} \nabla_{x}\left(p-\frac{P^{\mathrm{e}}}{T^{\mathrm{e}}} \theta\right) \\
F_{x y}(p, \theta)= & F^{0}(p, \theta)+\epsilon F^{1}(p)+\epsilon^{2} F^{2}(p) \\
& F^{0}(p, \theta)=i \omega p-i \omega \rho^{\mathrm{e}} c_{p} \theta+\kappa \triangle_{y}(\theta) \\
& F^{1}(\theta)=2 \kappa \triangle_{y x}(\theta) \\
& F^{2}(\theta)=\kappa \triangle_{x}(\theta)
\end{aligned}
$$

Introduce now the expansions (8) of $p, v$, and $\theta$ in $G_{x y}, N_{x y}$, and $F_{x y}$ and identify terms of identical power of $\epsilon$. These balance equations become series in power of $\epsilon$ identically equal to zero whatever $\epsilon \ll 1$, so that each term must vanish. The so-derived equations at each order-combined with the adherence and isothermal conditions on $\Gamma$ satisfied by each ${ }^{i} v$ and ${ }^{i} \theta$-lead to a series of problems to be solved recurrently.

\subsection{Homogenized Description}

\subsubsection{Macroscopic Mass Balances}

The integration of the mass balance of order $i \geq 0$ over the pore cell volume, provides:

$$
\int_{\Omega_{f}}\left\{\operatorname{div}_{y}\left({ }^{i+1} v\right)+\operatorname{div}_{x}\left({ }^{i} v\right)+i \omega\left(\frac{{ }^{i} p}{P^{\mathrm{e}}}-\frac{{ }^{i} \theta}{T^{\mathrm{e}}}\right)\right\} \mathrm{d} \Omega=0
$$

From the divergence theorem, the periodicity and the adherence condition on $\Gamma$, the first term vanishes. Then, inverting $y$-integration and $x$-derivative and introducing the physical (i.e., 
observable) macro-variables:

$$
{ }^{i} V=\frac{1}{\Omega} \int_{\Omega_{\mathrm{f}}} \epsilon^{i} v \mathrm{~d} \Omega=\frac{\phi}{\Omega_{\mathrm{f}}} \int_{\Omega_{\mathrm{f}}} \epsilon^{i i} v \mathrm{~d} \Omega ; \quad{ }^{i} P=\frac{1}{\Omega_{\mathrm{f}}} \int_{\Omega_{\mathrm{f}}} \epsilon^{i i} p \mathrm{~d} \Omega ; \quad{ }^{i} T=\frac{1}{\Omega_{\mathrm{f}}} \int_{\Omega_{\mathrm{f}}} \epsilon^{i i} \theta \mathrm{d} \Omega .
$$

one obtains the macroscopic mass balance at order $i$ :

$$
\operatorname{div}_{x}\left({ }^{i} V\right)+i \omega \phi\left(\frac{{ }^{i} P}{P^{\mathrm{e}}}-\frac{{ }^{i} T}{T^{\mathrm{e}}}\right)=0
$$

To go further, we need to determine the local fields $\left({ }^{i} p,{ }^{i} v,{ }^{i} \theta\right)$ by solving successively the harmonic visco-thermal linear problems in the cell. The first steps of the resolution are recalled hereafter [for more details see (Boutin 2007)].

\subsubsection{Pressure and Temperature at the Leading (Zero) Order}

The momentum balance at order $\epsilon^{-1}$ reduces to $N^{-1}\left({ }^{0} p\right)=-\nabla_{y}{ }^{0} p=0$ that corresponds to a degenerated $P$-mode, i.e., a static regime of pressure ${ }^{0} p$ at pores scale:

$$
{ }^{0} p(x, y)={ }^{0} P(x)
$$

This result introduced in the heat balance at order $\epsilon^{0}, F^{0}\left({ }^{0} P,{ }^{0} \theta\right)=0$ leads to the differential system $\left\{S_{t}^{0}\right\}$ set on $\Omega_{\mathrm{f}}$, governing ${ }^{0} \theta$. The presence of the thermal inertia term clearly indicates the transient thermal regime of the $T$-mode at pores scale:

$$
S_{t}^{0}\left\{\begin{array}{l}
i \omega^{0} P(x)-i \omega \rho^{\mathrm{e}} c_{p}{ }^{0} \theta+\kappa \triangle_{y}\left({ }^{0} \theta\right)=0 \\
{ }^{0} \theta / \Gamma=0 ; \quad{ }^{0} \theta \Omega-\text { periodic }
\end{array}\right.
$$

The solution of this linear problem, with ${ }^{0} P(x)$ as forcing term, reads:

$$
\frac{{ }^{0} \theta(x, y)}{T^{\mathrm{e}}}={ }^{0} \pi\left(\frac{y}{\delta_{t}}\right) \frac{{ }^{0} P(x)}{P^{\mathrm{e}}}
$$

The temperature distribution ${ }^{0} \pi$ (solution for ${ }^{0} P(x)=\frac{P^{\mathrm{e}}}{T^{\mathrm{e}}}$ ) is complex and depends on the local variable and the frequency through the dimensionless variable $\frac{y}{\delta_{t}}$. In isothermal regime, reached at low frequency $\left(\frac{\ell}{\delta_{t}} \rightarrow 0\right)$, the temperature vanishes, ${ }^{0} \pi \rightarrow 0$. In adiabatic regime, reached at high frequency $\left(\frac{\ell}{\delta_{t}} \rightarrow \infty\right)$, the temperature tends to the uniform value $\frac{P^{\mathrm{e}}}{\rho^{\mathrm{e}} c_{p} T^{\mathrm{e}}}=(1-1 / \gamma)$.

\subsubsection{Local Velocity, Pressure, and Temperature at the First Order}

The local velocity ${ }^{0} v$, and the pressure of the first order ${ }^{1} p$, are derived from the system set on $\Omega_{\mathrm{f}}$ :

$$
S_{v}^{0}\left\{\begin{array}{c}
-\nabla_{y}{ }^{1} p-\nabla_{x}{ }^{0} P-i \omega \rho^{\mathrm{e}}{ }^{0} v+\triangle_{y}\left(\mu^{0} v\right)=0 \\
\operatorname{div}_{y}\left({ }^{0} v\right)=0 \\
{ }^{0} v_{/ \Gamma}=0 ; \quad{ }^{0} v \quad \& \quad{ }^{1} p \quad \Omega \text { - periodic }
\end{array}\right.
$$

The (partial) balance of the viscous forces by the inertia of the gas expresses the dynamic regime of the $S$-mode at pores scale. Taking the divergence of $\left\{S_{v}^{0}\right\}_{a}$ gives $\triangle_{y}{ }^{1} p=0$, hence 
the $P$-mode again degenerates into a static regime at this order. Set $\left\{S_{v}^{0}\right\}$ defines the linear dynamic permeability problem with $\nabla_{x}^{0} P$ as forcing term, whose solution is, Auriault (1980):

$$
\mu^{0} v(x, y)={ }^{0} k\left(\frac{y}{\delta_{v}}\right) \cdot \nabla_{x}{ }^{0} P ; \quad{ }^{1} p(x, y)={ }^{1} \alpha\left(\frac{y}{\delta_{v}}\right) \cdot \nabla_{x}{ }^{0} P+\widehat{{ }^{1} p}(x)
$$

The tensors ${ }^{0} k$ and ${ }^{1} \alpha$ are constituted by velocity and pressure (of zero mean value on $\Omega_{\mathrm{f}}$ ) $\left\{-{ }^{0} k^{i} / \mu,{ }^{1} \alpha^{i}\right\}$ corresponding to unit pressure gradient, $\nabla_{x}{ }^{0} P(x)=\mathrm{e}_{i}$. The fields $\left\{{ }^{0} k^{i},{ }^{1} \alpha^{i}\right\}$ are complex and depend on the dimensionless variable $\frac{y}{\delta_{v}}$. At low frequency $\left(\frac{l}{\delta_{v}} \rightarrow 0\right)$, the inertial effect vanishes and ${ }^{0} k$ tends to be real. At high frequency $\left(\frac{l}{\delta_{v}} \rightarrow \infty\right)$, the inertia dominates and ${ }^{0} k$ tends to a pure imaginary tensor.

\subsection{Classical Poro-acoustic Modeling}

Reporting the local fields in the zero-order global mass balance (17) leads to the macroscopic description at the leading order. The equivalent continuum is in agreement with the phenomenological approaches of Zwikker and Kosten (1949), Attenborough (1983), and Allard (1993) (in the sequel, the index ${ }_{x}$ is omitted for the macro derivatives):

$$
\begin{aligned}
\operatorname{div}\left({ }^{0} V\right)+i \omega \phi\left[\frac{{ }^{0} P}{P^{\mathrm{e}}}-\frac{{ }^{0} T}{T^{\mathrm{e}}}\right]=0 & \\
\mu^{0} V=-{ }^{0} K \cdot \nabla{ }^{0} P ; & { }^{0} K=\frac{\phi}{\Omega_{f}} \int_{\Omega_{\mathrm{f}}}{ }^{0} k \mathrm{~d} \Omega \\
\frac{{ }^{0} T}{T^{\mathrm{e}}}={ }^{0} \Pi \frac{{ }^{0} P}{P^{\mathrm{e}}} ; & { }^{0} \Pi=\frac{1}{\Omega_{\mathrm{f}}} \int_{\Omega_{\mathrm{f}}}{ }^{0} \pi \mathrm{d} \Omega .
\end{aligned}
$$

The dynamic permeability tensor ${ }^{0} K / \mu$ and the effective compressibility $\phi\left[1-{ }^{0} \Pi\right] / P^{\mathrm{e}}$, depend on the frequency. At low frequencies, (i) ${ }^{0} K$ tends toward the real-valued intrinsic permeability $\mathcal{K}=O\left(\ell^{2}\right)$, (ii) ${ }^{0} \Pi \rightarrow 0$ and the effective compressibility tends toward its isothermal value $\phi / P^{\mathrm{e}}$ (more precisely $\frac{{ }^{0} \Pi}{i \omega} \frac{T^{\mathrm{e}}}{P^{\mathrm{e}}} \rightarrow \Upsilon / \kappa, \Upsilon$ being referred to thermal permeability $\left(O\left(\ell^{2}\right)\right)$ by analogy with the intrinsic permeability, (Champoux and Allard 1991). At high frequencies, (i) ${ }^{0} K$ tends toward a pure imaginary value, $\frac{\phi \mu}{i \omega \rho^{\alpha} \alpha_{\infty}}$ where $\alpha_{\infty}$ is the tortuosity, (ii) the perturbations become quasi-adiabatic so that ${ }^{0} \Pi \rightarrow 1-1 / \gamma$, and the compressibility tends toward $\phi / \gamma P^{\mathrm{e}}$. Low- and high-frequency domains of shear and thermal phenomena are, respectively, delimited by critical frequencies $\omega_{v}$ and $\omega_{t}$ derived by equalizing low- and high-frequency effects:

$$
\omega_{v}=\frac{\phi \mu}{\mathcal{K} \rho^{\mathrm{e}} \alpha_{\infty}} ; \quad \omega_{t}=\frac{\kappa}{\Upsilon \rho^{\mathrm{e}} c_{p}}
$$

3.4 Space Locality and Time Non-locality

The description (20) is local in space in the sense that, on a given point, the flux (resp. mean temperature) is related to the pressure gradient (resp. pressure) on this point.

Besides, the modeling is non-local in time. This relies on the frequency dependance of the $S$ - and $T$-modes involved in the effective parameters ${ }^{0} K\left(\omega / \omega_{v}\right)$ and ${ }^{0} \Pi\left(\omega / \omega_{t}\right)$. Consider for instance the dynamic Darcy's law. Coming back in the time domain (inverse Fourier 
transform and convolution product are, respectively, denoted by * and $*$ ), it reads Auriault (1980):

$$
\mu \widehat{{ }^{0} V}(t)=-{ }^{0} K^{*}\left(\frac{\tau}{\tau_{v}}\right) * \nabla^{0} P^{*}=-\int_{-\infty}^{\infty}{ }^{0} K^{*}\left(\frac{\tau}{\tau_{v}}\right) \cdot \nabla^{0} P^{*}(t-\tau) d \tau
$$

Therefore, the flux at a given time is not related to the instantaneous pressure gradient, but to the history of the pressure gradient (on a duration $\tau_{v}=O\left(1 / \omega_{v}\right)$ characteristic of the $S$-mode). Expanding $\nabla^{0} P^{*}$ in Taylor series around $t$ and noticing that $\int_{0}^{\infty}{ }^{0} K^{*}\left(\frac{\tau}{\tau_{v}}\right) d \tau=$ ${ }^{0} K(0)=\mathcal{K}$, one obtains:

$$
\begin{aligned}
\mu^{0} V^{*}(t)= & -\mathcal{K} . \nabla^{0} P^{*}(t)+\left[\int_{0}^{\infty}{ }^{0} K^{*}\left(\frac{\tau}{\tau_{v}}\right) \tau d \tau\right] \cdot \frac{\partial}{\partial t}\left[\nabla^{0} P^{*}(t)\right] \\
& -\left[\int_{0}^{\infty}{ }^{0} K^{*}\left(\frac{\tau}{\tau_{v}}\right) \tau^{2} d \tau\right] \cdot \frac{\partial^{2}}{\partial t^{2}}\left[\nabla^{0} P^{*}(t)\right]+\cdots
\end{aligned}
$$

Thus, the dynamic Darcy's law is local in time when the time derivatives of the pressure gradient vanish, i.e., in stationary regime. By construction, the $i$ th term of the expansion of the convolution is $O\left(\mathcal{K}\left(\frac{\tau_{v}}{t}\right)^{i} \nabla^{0} P^{*}\right)=O\left(\mathcal{K}\left(\frac{\omega}{\omega_{v}}\right)^{i} \nabla^{0} P\right)$, hence for frequency sufficiently low, $\omega / \omega_{v} \ll 1$, the dynamic Darcy's law is "slightly" non-local in time. Note that, in this case, the $S$-mode regime at pores scale (set $\left\{S_{v}^{0}\right\}$ ) degenerate into a quasi-static regime.

Similar remarks apply for the mean temperature that presents a memory effect—of duration $\tau_{t}=O\left(1 / \omega_{t}\right)$ characteristic of the $T$-mode-as indicated by the macroscopic law expressed in the time domain:

$$
\begin{aligned}
\frac{{ }^{0} T^{*}(t)}{T^{\mathrm{e}}}= & \int_{0}^{\infty}{ }_{0}^{0} \Pi^{*}\left(\frac{\tau}{\tau_{t}}\right) \frac{{ }^{0} P^{*}(t-\tau)}{P^{\mathrm{e}}} \mathrm{d} \tau=\frac{\Upsilon}{\kappa T^{\mathrm{e}}} \frac{\partial}{\partial t}{ }^{0} P^{*}(t) \\
& -\left[\int_{0}^{\infty}{ }^{0} \Pi^{*}\left(\frac{\tau}{\tau_{t}}\right) \tau^{2} \mathrm{~d} \tau\right] \frac{\partial^{2}}{\partial t^{2}} \frac{{ }^{0}}{P^{*}(t)} P^{\mathrm{e}}+\cdots
\end{aligned}
$$

This shows that the macro non-locality or memory effects arise from the transient regime of the $T$ - and $S$-modes at the ERV scale. In the same frequency range, the $P$-mode at pores scale remains in quasi-static regime, hence the pressure varies at large scale. This enables to respect the requirement of a scale separation and to derive the macro behavior. At sufficiently low frequency (strictly zero frequency) the local regime of the three $T$-, $S$-, $P$-modes tends to be stationary and the time non-locality tends to disappear.

\subsection{Local and Global Modes}

From the three modes at pores scale, the up-scaling leads to a single macroscopic acoustic mode. Clearly the $S$ - and $T$-modes of wavelength of the order of the pores size disappear at the macroscale, but their effects are included in the macro-parameters. The wave equation derived by eliminating the flux and the temperature:

$$
-\operatorname{div}\left(\frac{{ }^{0} K}{\mu} \cdot \nabla^{0} P\right)+i \omega \frac{\phi\left[1-{ }^{0} \Pi\right]}{P^{e}}{ }^{0} P=0
$$


evidences that the $P$-mode (of the gas in the absence of solid), associated to pressure varying at large scale, (quasi-static regime at pores scale) is drastically modified into a Biot wave of $P_{2}$ type. The $P_{2}$ waves features can be identified from harmonic plane waves:

$$
{ }^{0} P=\left|{ }^{0} P_{0}\right| \exp (-i h x) \exp (i \omega t)
$$

Assuming isotropy for simplicity, $\left({ }^{0} K={ }^{0} \underline{K} I\right)$ the complex wave number $h=h_{r}+i h_{i}$ is given by:

$$
(-i h)^{2}=i \omega \frac{\phi\left[1-{ }^{0} \Pi\right]}{P^{\mathrm{e}}} \frac{\mu}{\underline{{ }^{0} K}}
$$

Thus, the wave velocity $C$, wavelength $\Lambda$, and attenuation $\xi$ read:

$$
h=\frac{\omega}{C}(1-i \xi), \quad \text { i.e., } \quad C=\frac{\omega}{h_{r}} ; \quad \frac{\Lambda}{2 \pi}=\frac{1}{h_{r}}=O\left(\left|\sqrt{\frac{{ }^{0} \underline{K}}{i \omega \mu \phi} \frac{P^{\mathrm{e}}}{1-{ }^{0} \Pi}}\right|\right) ; \quad \xi=-\frac{h_{i}}{h_{r}}
$$

Consequently, the $S$ - and $T$-modes in transient local regime, result in a strong dispersion of the $P_{2}$ wave, evolving from:

- a diffusion wave at low frequency $\left(\omega<\omega_{c}\right): C \simeq \sqrt{\frac{\omega}{\omega_{c}}} \sqrt{\frac{P^{\mathrm{e}}}{\alpha_{\infty} \rho^{\mathrm{e}}}} ; \xi_{d}=1-O\left(\sqrt{\frac{\omega}{\omega_{c}}}\right)$;

- to a propagation wave at high frequency $\left(\omega>\omega_{c}\right): C \simeq \sqrt{\frac{\gamma P^{\mathrm{e}}}{\alpha_{\infty} \rho^{\mathrm{e}}}} ; \xi_{d} \sim \sqrt{\frac{\omega_{c}}{\omega}}$.

Those results at the leading order are derived with a degenerated local $P$-mode in static regime. In the next section, a situation with weak inner dynamics of the $P$-mode is examined.

\section{Poro-acoustics with Scattering}

Rayleigh scattering of sound in porous media occurs in the case of poor scale separation, i.e., when the wavelength is large but not too large compared to the ERV size. Only few studies focus on this topic, e.g., Tournat et al. (2004). To describe this situation, the homogenization method still applies, however, the procedure generally restrained to the derivation of the leading order, has to be carried on to the next orders (Boutin and Auriault 1993; Boutin 2007). Such a poro-acoustic description, enriched by the first and second-order terms, has been derived in Boutin (2007). Here, we only indicate the process of resolution of higher order problems. The macro-description enhanced up to the second-order accounts for "slight dynamics" of the $P$-mode at pores scale and the influence of this phenomenon is investigated.

\subsection{Local Fields at the Two Next Orders}

Following the process of successive resolution, the first corrector ${ }^{1} \theta$ of the temperature is derived from the heat transfer at order $\epsilon$ governed by the differential system $\left\{S_{t}^{1}\right\}$ set on $\Omega_{f}$ :

$$
S_{t}^{1}\left\{\begin{array}{l}
i \omega^{1} p(x, y)-i \omega \rho^{\mathrm{e}} c_{p}{ }^{1} \theta+\kappa \triangle_{y}\left({ }^{1} \theta\right)+2 \kappa \triangle_{y x}\left({ }^{0} \theta\right)=0 \\
{ }^{1} \theta_{/ \Gamma}=0 ; \quad{ }^{1} \theta \quad \Omega \text { - periodic }
\end{array}\right.
$$

The previously determined fields ${ }^{0} \theta$ and ${ }^{1} p$ (38 and 19) introduce two-independent forcing terms:

- $\widehat{{ }^{1} p}(x)$, inducing an identical problem than $\left(S_{t}^{0}\right)$ except that $\widehat{{ }^{1} p}(x)$ replaces ${ }^{0} P(x)$,

- $\nabla_{x}{ }^{0} P$, linked with ${ }^{1} \alpha$ and $\nabla_{y}{ }^{0} \pi$ (coming from the equality: $\triangle_{y x}\left({ }^{0} \theta\right)=\frac{T^{\mathrm{e}}}{P^{\mathrm{e}}} \nabla_{y}^{0} \pi \cdot \nabla_{x}{ }^{0} P$ ). 
Therefore, by linearity, the solution is in the form:

$$
\frac{{ }^{1} \theta(x, y)}{T^{\mathrm{e}}}={ }^{0} \pi\left(\frac{y}{\delta_{t}}\right) \frac{\widehat{{ }^{1} p}(x)}{P^{\mathrm{e}}}+{ }^{1} \pi\left(\frac{y}{\delta_{t}}, \frac{y}{\delta_{v}}\right) \cdot \frac{\nabla_{x}{ }^{0} P}{P^{\mathrm{e}}}
$$

The tensor of temperature distribution ${ }^{1} \pi$ is constituted by complex and frequency-dependent solutions ${ }^{1} \pi^{i}$ corresponding to pressure gradient, $\nabla_{x}{ }^{0} P(x)=\frac{P^{\mathrm{e}}}{T^{\mathrm{e}}} \mathrm{e}_{i}$. This corresponds to a transient regime with thermo-viscous coupling.

Similarly, the pressure and velocity, ${ }^{2} p$ and ${ }^{1} v$, are determined from the set $\left\{S_{v}^{1}\right\}$ :

$$
S_{v}^{1}\left\{\begin{array}{l}
N^{-1}\left({ }^{2} p\right)+N^{0}\left({ }^{1} p,{ }^{1} v\right)+N^{1}\left({ }^{0} P,{ }^{0} v,{ }^{0} \theta\right)=0 \\
G^{-1}\left({ }^{1} v\right)+G^{0}\left({ }^{0} P,{ }^{0} v,{ }^{0} \theta\right)=0 \\
{ }^{1} v_{/ \Gamma}=0 ;{ }^{1} v \quad \& \quad{ }^{2} p \quad \Omega \text { - periodic }
\end{array}\right.
$$

the temperature ${ }^{2} \theta$ from the set $\left\{S_{t}^{2}\right\}$ :

$$
S_{t}^{2}\left\{\begin{array}{l}
F^{0}\left({ }^{2} p,{ }^{2} \theta\right)+F^{1}\left({ }^{1} \theta\right)+F^{2}\left({ }^{0} \theta\right)=0 \\
{ }^{2} \theta / \Gamma=0 ; \quad{ }^{2} \theta \quad \Omega \text { - periodic }
\end{array}\right.
$$

and ${ }^{3} p$ and ${ }^{2} v$ are derived from $\left\{S_{v}^{2}\right\}$ :

$$
S_{v}^{2}\left\{\begin{array}{l}
N^{-1}\left({ }^{3} p\right)+N^{0}\left({ }^{2} p,{ }^{2} v\right)+N^{1}\left({ }^{1} p,{ }^{1} v,{ }^{1} \theta\right)+N^{2}\left({ }^{0} P,{ }^{0} v,{ }^{0} \theta\right)=0 \\
G^{-1}\left({ }^{2} v\right)+G^{0}\left({ }^{1} p,{ }^{1} v,{ }^{1} \theta\right)=0 \\
{ }^{2} v_{/ \Gamma}=0 ;{ }^{2} v \text { and }{ }^{3} p \quad \Omega \text { - periodic }
\end{array}\right.
$$

At each order, the same differential set as the previous order is recovered, except for new additive terms, involving the gradient of the solutions of lower order. Thus, once $\left({ }^{j+1} p,{ }^{j} v,{ }^{j} \theta\right)$, $0 \leq j<i$, have been determined, they become forcing terms in the problems related to $\left({ }^{i+1} p,{ }^{i} v,{ }^{i} \theta\right)$, and so on.... For this reason, conversely to the previous orders, the set $\left\{S_{v}^{1}\right\}$ (a fortiori $\left\{S_{v}^{2}\right\}$ ) describes an "out of equilibrium" state for the pressure in the period $\left(\triangle_{y}{ }^{2} p \neq 0\right.$,) that reveals a slight inner dynamics for the $P$-mode at the pore scale. Finally, up to the second, the local fields reads:

$$
\begin{aligned}
p(x, y)= & { }^{0} P(x)+\epsilon\left[\widehat{{ }^{1} p}(x)+{ }^{1} \alpha \nabla_{x}{ }^{0} P(x)\right]+\epsilon^{2}\left[\widehat{{ }^{2} p}(x)+{ }^{1} \alpha \nabla_{x} \widehat{{ }^{1} p}(x)\right. \\
& \left.+{ }^{2} \alpha . . \nabla_{x} \nabla_{x}{ }^{0} P(x)+\frac{i \omega \mu}{P^{\mathrm{e}}}{ }^{0}{ }^{0} P(x)\right]+\cdots \\
-\mu v(x, y)= & { }^{0} k \cdot \nabla_{x}{ }^{0} P(x)+\epsilon\left[{ }^{0} k \cdot \nabla_{x} \widehat{{ }^{1} p}(x)+{ }^{1} k . . \nabla_{x} \nabla_{x}{ }^{0} P(x)+\frac{i \omega \mu}{P^{e}}{ }^{1} n^{0} P(x)\right] \\
& +\epsilon^{2}\left[{ }^{0} k \cdot \nabla_{x} \widehat{{ }^{2} p}(x)+{ }^{1} k . . \nabla_{x} \nabla_{x} \widehat{{ }^{1} p}(x)+{ }^{2} k \ldots \nabla_{x} \nabla_{x} \nabla_{x}{ }^{0} P(x)\right. \\
& \left.+\frac{i \omega \mu}{P^{\mathrm{e}}}{ }^{2} n \cdot \nabla_{x}{ }^{0} P(x)\right]+\cdots \\
\left(P^{\mathrm{e}} / T^{\mathrm{e}}\right) \theta(x, y)= & { }^{0}{ }^{0} P(x)+\epsilon\left[{ }^{0} \pi \widehat{{ }^{1} p}(x)+{ }^{1} \pi \cdot \nabla_{x}{ }^{0} P(x)\right]+\epsilon^{2}\left[{ }^{0} \pi \widehat{{ }^{2} p}(x)+{ }^{1} \pi . \nabla_{x} \widehat{{ }^{1} p}(x)\right. \\
& \left.+{ }^{2} \pi . . \nabla_{x} \nabla_{x}{ }^{0} P(x)+\frac{i \omega \mu}{P^{\mathrm{e}}} \zeta^{0} P(x)\right]+\cdots
\end{aligned}
$$

where:

- $\left(-{ }^{1} k^{m n} / \mu,{ }^{2} \alpha^{m n}\right)$ and $\left(-{ }^{1} n / \mu,{ }^{2} v\right)$, are the solutions of $\left(S_{v}^{1}\right)$ with $\nabla_{x} \nabla_{x}{ }^{0} P(x)=\mathrm{e}_{\mathrm{m}} \otimes$ $\mathrm{e}_{n}$, and with $\frac{i \omega \mu}{P^{\mathrm{e}}}{ }^{0} P(x)=1$, respectively.

- $\left(-{ }^{2} k^{p q r} / \mu,{ }^{3} \alpha^{p q r}\right)$ and $\left(-{ }^{2} n^{m} / \mu,{ }^{3} v^{m}\right)$, are the solutions of $\left(S_{v}^{2}\right)$ with $\nabla_{x} \nabla_{x} \nabla_{x}{ }^{0} P(x)=$ $\mathrm{e}_{p} \otimes \mathrm{e}_{q} \otimes \mathrm{e}_{r}$ and with $\frac{i \omega \mu}{P^{\mathrm{e}}} \nabla_{x}{ }^{0} P(x)=\mathrm{e}_{\mathrm{m}}$, respectively. 
- ${ }^{i} \pi$ are the solutions of $\left(S_{t}^{i}\right)$ under unit component of $\frac{T^{\mathrm{e}}}{P^{\mathrm{e}}}\left(\nabla_{x}\right){ }^{i}{ }^{0} P$ and ${ }^{2} \zeta$ is the solution of $\left(S_{t}^{2}\right)$ under $\frac{i \omega \mu}{P^{\mathrm{e}}}{ }^{0} P(x)=1$.

\subsection{Enhanced Poro-acoustics}

The mean value of the local fields up to the second-order:

$$
\begin{aligned}
& P(x)=\frac{1}{\Omega} \int_{\Omega_{\mathrm{f}}} p(x, y) \mathrm{d} \Omega={ }^{0} P(x)+{ }^{1} P(x)+{ }^{2} P(x) \\
& V(x)=\frac{1}{\Omega} \int_{\Omega_{\mathrm{f}}} v(x, y) \mathrm{d} \Omega={ }^{0} V(x)+{ }^{1} V(x)+{ }^{2} V(x) \\
& T(x)=\frac{1}{\Omega} \int_{\Omega_{\mathrm{f}}} \theta(x, y) \mathrm{d} \Omega={ }^{0} T(x)+{ }^{1} T(x)+{ }^{2} T(x)
\end{aligned}
$$

provides the macro-variables appearing in the macro mass balances (17) and the macroscopic tensors ${ }^{i} K \sim O\left({ }^{0} K\right) l^{i},{ }^{i} \Pi \sim l^{i},{ }^{i} N \sim l^{i}$, and ${ }^{2} Z \sim O(1)$ (the local fields of pressure $\alpha^{i}, v^{i}$ are of zero mean value):

${ }^{i} K=\frac{1}{\Omega} \int_{\Omega_{\mathrm{f}}}{ }^{i} k \epsilon^{i} \mathrm{~d} \Omega ; \quad{ }^{i} \Pi=\frac{1}{\Omega_{\mathrm{f}}} \int_{\Omega_{\mathrm{f}}}{ }^{i} \pi \epsilon^{i} \mathrm{~d} \Omega ; \quad{ }^{i} N=\frac{1}{\Omega} \int_{\Omega_{\mathrm{f}}}{ }^{i} n \epsilon^{i} \mathrm{~d} \Omega ; \quad{ }^{2} Z=v \frac{1}{\Omega_{\mathrm{f}}} \int_{\Omega_{\mathrm{f}}}{ }^{2} \zeta \epsilon^{2} \mathrm{~d} \Omega$

The sum of the mass balances Eq. (17) at the leading and at the two next orders, achieves the poro-acoustics description (of third-order accuracy) in the presence of poor scale separation:

$$
\begin{aligned}
& \operatorname{div}(V)+i \omega \phi\left[\frac{P}{P^{\mathrm{e}}}-\frac{T}{T^{\mathrm{e}}}\right]=0 \\
& \mu V=-{ }^{0} K . \nabla P-{ }^{1} K . . \nabla \nabla P-{ }^{2} K \ldots \nabla \nabla \nabla P-\frac{i \omega \mu}{P^{\mathrm{e}}}{ }^{1} N P-\frac{i \omega \mu}{P^{\mathrm{e}}}{ }^{2} N . \nabla P \\
& \frac{T}{T^{\mathrm{e}}}={ }^{0} \Pi \frac{P}{P^{\mathrm{e}}}+{ }^{1} \Pi . \nabla \frac{P}{P^{\mathrm{e}}}+{ }^{2} \Pi . . \nabla \nabla \frac{P}{P^{\mathrm{e}}}+\frac{i \omega \mu}{P^{\mathrm{e}}}{ }^{2} Z \frac{P}{P^{\mathrm{e}}}
\end{aligned}
$$

This description could be extended to higher order terms. The general formulation will be completed by similar additional correctors associated to higher gradients of pressure.

Remark: A detailed analysis of the properties of the local fields enables to demonstrate that the first corrector of pressure ${ }^{1} P$ vanishes (Boutin 2007). This is obvious in case of macro-isotropy since odd rank tensors are necessarily null, hence ${ }^{1} \mathrm{P}$ vanishes (and also ${ }^{1} \mathrm{~V}$ and ${ }^{1} T$ in this case).

\subsection{Space and Time Non-locality of Correctors}

As for classical poro-acoustics, the enhanced poro-acoustics is non-local in time because of the frequency dependance of the macroscopic parameters at the several orders. As a consequence, the effective memory effects are modified ; however, (i) the characteristic frequencies are identical as at the leading order and (ii) no new characteristic memory time arises from the slight dynamics of the $P$-mode at pores scale.

The new point is that the description is also non-local in space: the correctors introduced by the poor scale separation introduces successive gradients in the enhanced dynamic Darcy's law and macroscopic thermal law. By construction, these terms are of weak magnitude $O\left([2 \pi \ell / \Lambda]^{i}\right)$ compared to the leading order. Thus, the poor scale separation induces 
a "weak non-locality in space". Strictly speaking, a pure locality in space would require $2 \pi \ell / \Lambda \rightarrow 0$, which may only be reached at stationary regime-also local in time-but not in acoustics.

The macroscopic laws can equivalently be though as the first terms of the expansion of a spacial convolution product (denoted by $\star$ ) of the local physical variables with non-local effective tensors (and functions) of the media (denoted by .), i.e.,:

$$
\begin{aligned}
& \mu V\left(x_{0}\right) \approx-\widetilde{K} \star \nabla P-\frac{i \omega \mu}{P^{\mathrm{e}}} \widetilde{N} \star P=\int_{R^{3}} \widetilde{K}(x) . \nabla P\left(x_{0}-x\right) \mathrm{d} x-\frac{i \omega \mu}{P^{\mathrm{e}}} \int_{R^{3}} \widetilde{N}(x) P\left(x_{0}-x\right) \mathrm{d} x \\
& \frac{P^{\mathrm{e}}}{T^{\mathrm{e}}} T\left(x_{0}\right) \approx \widetilde{\Pi} \star P+\frac{i \omega \mu}{P^{\mathrm{e}}} \widetilde{Z} \star \frac{P}{P^{\mathrm{e}}}=\int_{R^{3}} \widetilde{\Pi}(x) P\left(x_{0}-x\right) \mathrm{d} x-\frac{i \omega \mu}{P^{\mathrm{e}}} \int_{R^{3}} \widetilde{Z}(x) P\left(x_{0}-x\right) \mathrm{d} x
\end{aligned}
$$

The tensors ${ }^{i} K$, derived by homogenization are related to the non-local complex tensor $\widetilde{K}(x)$ by the identities:

$$
{ }^{0} K=\int_{R^{3}} \widetilde{K}(x) \mathrm{d} x ; \quad{ }^{1} K=\int_{R^{3}} \widetilde{K}(x) \otimes x \mathrm{~d} x ; \quad{ }^{2} K=\int_{R^{3}} \widetilde{K}(x) \otimes x \otimes x \mathrm{~d} x
$$

and similarly for the non-local functions $\widetilde{\Pi}, \widetilde{N}, \widetilde{Z}$ :

$$
{ }^{i} \Pi=\int_{R^{3}} \widetilde{\Pi}(x)(\otimes x)^{i} \mathrm{~d} x ; \quad{ }^{i+1} N=\int_{R^{3}} \widetilde{N}(x)(\otimes x)^{i} \mathrm{~d} x \quad ; \quad{ }^{i+2} Z=\int_{R^{3}} \widetilde{Z}(x)(\otimes x)^{i} \mathrm{~d} x
$$

In this framework, local laws would correspond to $\widetilde{K}(x)={ }^{0} K \delta(x)$; $\widetilde{\Pi}(x)={ }^{0} \Pi \delta(x)$, etc... (where $\delta(x)$ is the 3D-Dirac distribution). In fact, the high order terms established by homogenization show that the spacial extension of the non-local effective dynamic permeability $\widetilde{K}(x)$ is of the order of the cell size $\ell$. Because of the requirement of scale separation, the complete determination of the non-local effective parameters is not necessary (the relevant scale of spacial variation is the cell size).

The origin of the correctors lies in the terms neglected at preceding orders that become significant at the considered order. For simplicity, consider macro-isotropy, then the odd tensors vanish and the even tensors, applied to the successive pressure gradients, can be reduced to scalar functions (denoted, ${ }^{0} K,{ }^{2} K$, etc....). With an accuracy up to the third order, the macroscopic laws can equivalently be rewritten as:

$$
\begin{aligned}
\mu\left[V-\frac{\underline{K}}{{ }^{0} \underline{K}} \Delta(V)\right] & =-{ }^{0} \underline{K}\left[1+\frac{i \omega \mu}{P^{\mathrm{e}}} \frac{\underline{N}}{{ }^{0} \underline{K}}\right] \nabla P \\
\frac{1}{T^{\mathrm{e}}}\left[T-\frac{{ }^{2} \underline{\Pi}}{{ }^{0} \underline{\Pi}} \triangle(T)\right] & ={ }^{0} \underline{\Pi}\left[1+\frac{i \omega \mu}{P^{\mathrm{e}}} \frac{\underline{Z}}{{ }^{0} \underline{\Pi}}\right] \frac{P}{P^{\mathrm{e}}}
\end{aligned}
$$

The dynamic Darcy law and thermal law are enriched by two terms:

- on the right hand side, the actual non-local corrector (in space and time) induced by the non-uniformity of the variable ( $V$ and $T$, hence $\nabla P$ ). As for Darcy's law, it corresponds to a generalization of the Brinkman's law in the dynamic range,

- on the left hand side, at this order, a local corrector in space (non-local in time) induced by the gas compressibility (negligible at the zero-order) with thermo-viscous coupling. In the Darcy's law, it expresses the weak contribution of the isotropic viscous stresses. 
In acoustics, the non-locality in space combines with non-locality in time. This comes from the spacial non-homogeneity of the fields that oscillate according to the frequency-dependent wavelength. However, as the spacial non-locality is intrinsically linked to the higher gradient, i.e., the non-homogeneity of the fields, this latter may also occurs in statics (on this point see Boutin 1996; Auriault et al. 2005).

\subsection{Space Non-locality and Eigen Modes with Rayleigh Scattering}

The space non-locality is closely related to the Rayleigh scattering that induces a correction of the zero-order eigen modes. This is established by investigating the second corrector driven by the set (in isotropic case):

$$
\begin{aligned}
& \operatorname{div}\left({ }^{2} V\right)+i \omega \phi\left[\frac{{ }^{2} P}{P^{\mathrm{e}}}-\frac{{ }^{2} T}{T^{\mathrm{e}}}\right]=0 \\
& \mu^{2} V=-{ }^{0} \underline{K} \nabla^{2} P-{ }^{2} \underline{K} \nabla \triangle^{0} P-\frac{i \omega \mu}{P^{\mathrm{e}}} \underline{N} \nabla^{0} P \\
& \frac{{ }^{2} T}{T^{\mathrm{e}}}={ }^{0} \underline{\Pi} \frac{{ }^{2} P}{P^{\mathrm{e}}}+{ }^{2} \underline{\Pi} \triangle \frac{{ }^{0} P}{P^{\mathrm{e}}}+\frac{i \omega \mu}{P^{\mathrm{e}}}{ }^{2} \underline{Z} \frac{{ }^{0} P}{P^{\mathrm{e}}}
\end{aligned}
$$

Thus, while the leading order of pressure ${ }^{0} P$ follows:

$$
-\frac{{ }^{0} \underline{K}}{\mu} \triangle^{0} P+i \omega \frac{\phi\left[1-{ }^{0} \underline{\Pi}\right]}{P^{\mathrm{e}}}{ }^{0} P=0
$$

the corrector ${ }^{2} P$ is driven by the same wave equation as ${ }^{0} P$ complemented by the source term $S^{2}\left({ }^{0} P\right)$ :

$$
-\frac{{ }^{0} \underline{K}}{\mu} \triangle^{2} P+i \omega \frac{\phi\left[1-{ }^{0} \underline{\Pi}\right]}{P^{\mathrm{e}}} 2 P=S^{2}\left({ }^{0} P\right)
$$

with:

$$
S^{2}\left({ }^{0} P\right)=\frac{{ }^{2} \underline{K}}{\mu} \triangle \triangle^{0} P+\frac{i \omega}{P^{\mathrm{e}}}\left[{ }^{2} \underline{N} \triangle^{0} P+\phi\left({ }^{2} \underline{\Pi} \triangle \triangle^{0} P+\frac{i \omega \mu}{P^{\mathrm{e}}} \underline{Z}^{2} P\right)\right]
$$

This is precisely the physics of the Rayleigh scattering: the passing of a long wave ${ }^{0} P$ through heterogeneities generates scattered sources $S^{2}\left({ }^{0} P\right)$ that radiate perturbations, i.e., the corrective ${ }^{2} P$ field. Considering a plane wave (23) at leading order, we have:

$$
S^{2}\left({ }^{0} P\right)=\left\{\frac{{ }^{2} \underline{K}}{\mu}(-i h)^{4}+\frac{i \omega}{P^{\mathrm{e}}}\left[{ }^{2} \underline{N}+{ }^{2} \underline{\Pi} \phi\right](-i h)^{2}+\mu \phi\left(\frac{i \omega}{P^{\mathrm{e}}}\right)^{2} \underline{\underline{Z}}\right\} \widetilde{{ }^{0} P} \exp (-i h x)
$$

so that the scattered source imposes a forcing to the medium according to its eigen mode itself. This induces a self-resonance effect at the macroscale, and subsequently ${ }^{2} P$ is linearly amplified as the wave progresses:

$$
\begin{aligned}
{ }^{2} P & =Q h^{2}(-i h x) \widetilde{\widetilde{0} P} \exp (-i h x) \quad \text { with } \\
2 Q & =\frac{{ }^{2} \underline{K}}{{ }^{0} \underline{K}}+\frac{{ }^{2} \underline{N}+{ }^{2} \underline{\Pi} \phi}{\phi\left(1-{ }^{0} \Pi\right)}+{ }^{2} \underline{Z} \phi \frac{{ }^{0} \underline{K}}{\left[\phi\left(1-{ }^{0} \underline{\Pi}\right)\right]^{2}}
\end{aligned}
$$

Finally, up to the second-order the macro-pressure reads:

$$
{ }^{0} P+{ }^{2} P=\left[1-Q(-i h)^{2}(-i h x)\right]^{\widetilde{0} P} \exp (-i h x)
$$


At the same level of approximation, within a distance $x$ such as $O(|h| x)<1 / \sqrt{\epsilon}$, the macro-pressure can also be expressed as:

$$
P=\widetilde{{ }^{0} P} \exp (-i \widetilde{h} x)+O\left(\epsilon^{3}\right) \quad \text { with } \quad \widetilde{h}=h\left[1+Q h^{2}\right]
$$

Thus, the diffraction modifies the zero-order wave number $h$ by a correction of the order of $Q h^{2}=O\left(\ell^{2} /(\lambda / 2 \pi)^{2}\right)$. This perturbation comes from the interference between the zeroorder wave and the coherent and amplified scattered wave. Owing to the dispersion of the $P_{2}$ wave, the perturbation of the wave celerity and attenuation are rather complex as discussed in Boutin (2007).

Note finally that the restriction to weak spacial non-locality, or equivalently to Rayleigh scattering, is imposed by the scale separation requirement. This exigence defines a frequency range that corresponds to weak transient effect of the $P$-mode at pores scale. In the next section, we examine the case where the $P$-mode actually reaches a dynamic regime at the local scale.

\section{Acoustics in Double Porosity Media}

From the first works of Barenblatt and ZheltovI (1960) and Warren and Root (1963), many studies have been devoted to double-porosity systems in geophysics. More recently, this topic has been addressed in acoustics, e.g., Only and Boutin (2003). Double-porosity media present a hierarchical morphology where the skeleton-that determines the pores $\Omega_{\mathrm{f}}$-is itself microporous (thus denoted $\Omega_{\mathrm{sm}}$ in the sequel). The micropores are characterized by a porosity $\phi_{\mathrm{m}}$ and a size $\ell_{\mathrm{m}}$ much smaller than the microporous domain size $d_{\mathrm{sm}}=O(\ell)$ and than the pores size $\ell$ (if not, the medium is of single porosity, of total porosity $\phi+(1-\phi) \phi_{\mathrm{m}}$ ). In the sequel, all the variables attached to the microporous domain are indexed by ${ }_{m}$ Fig. 2.

Such a morphology presenting two interconnected networks of very different characteristic sizes, enables a dynamic regime of the pressure within the microporous domain, that in turns, induces a deviation (non-local in time) from the macro behavior of single porosity media Boutin et al. (1998). The main physical points and the homogenization steps are summarized hereafter, then the macro influence of this additional non-local effect is discussed.

\subsection{Physical Analysis of Double-Porosity Media}

Three scales are then present in double-porosity media that can therefore be addressed by a three-scale homogenization, e.g., Auriault and Boutin (1994) and Boutin et al. (1998). However, the scale separation between pores and micropores enables to proceed by successive two-scale homogenizations - at least, when looking for a leading order description-namely a two-scale homogenization at the microscale to determine the properties of the microporous domain, then a second two-scale homogenization at pores scale, where the microporous domain is the equivalent homogeneous media derived in the previous step. This easier framework will be used here. Thus, the microporous domain is described by the single porosity media as exposed in Sect. 3. Since $\ell_{\mathrm{m}} \ll \ell$, the intrinsic permeability $\mathcal{K}_{\mathrm{m}}=O\left(\ell_{\mathrm{m}}^{2}\right)$ and the critical frequency $\omega_{v m}=\frac{\phi_{\mathrm{m}} \mu}{\mathcal{K}_{\mathrm{m}} \rho^{e} \alpha_{\infty \mathrm{m}}}$ of the microporous domain are much smaller (resp. larger) than the intrinsic permeability $\mathcal{K}=O\left(\ell^{2}\right)$ and the critical frequency $\omega_{v}=\frac{\phi \mu}{\mathcal{K} \rho^{e} \alpha_{\infty}}$ of the simple porous media (i.e., of single porosity $\phi$, neglecting the microporosity). This has two consequences: 


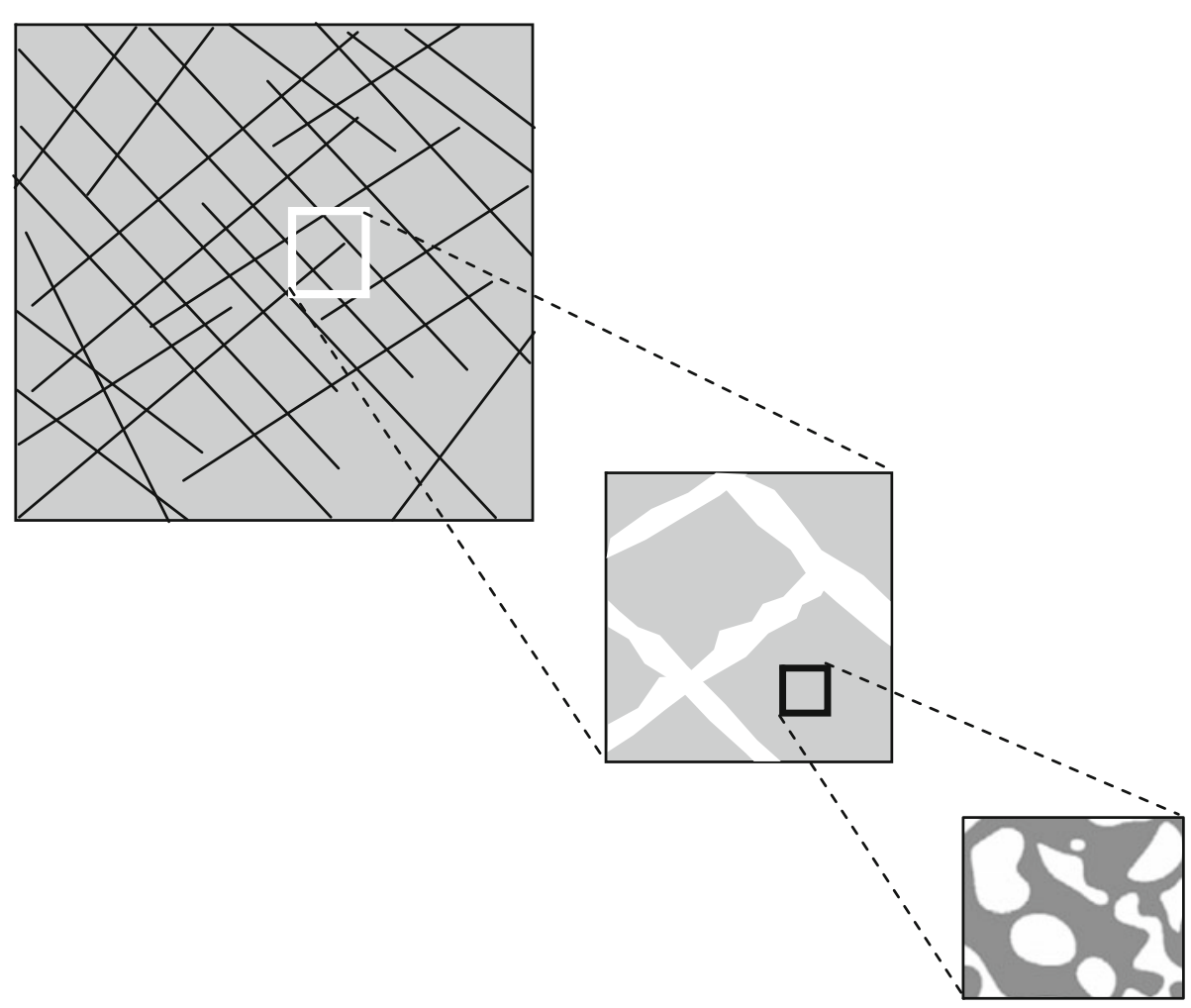

Fig. 2 Microstructure of double porosity media. Top Double porosity media. Middle cell of constituted by the pores $\Omega_{\mathrm{f}}$ of size $O(\ell)$ and the microporous domain $\Omega_{\mathrm{sm}}$ of size $O\left(d_{\mathrm{sm}}\right)=O(\ell)$. Bottom cell of the microporous domain of porosity $\phi_{\mathrm{m}}$ with pores of size $O\left(\ell_{\mathrm{m}}\right)$

- First, the flux in the micropores is significantly lesser than within the pores. For this reason, the influence of the micropores can only appear when the contrast of permeability is enough strong but not extremely large (if extremely large, the microporous domain is almost impervious and the medium reduces to single porosity, of porosity $\phi$ ). The weak contribution of the micropores insures that, in order of magnitude, the single porosity estimates applies for the global flow and the wavelength:

$$
\mu^{0} V=-O\left({ }^{0} K\right) \cdot \nabla^{0} P ; \quad \frac{\Lambda}{2 \pi}=O\left(\left|\sqrt{\frac{{ }^{0} K P^{\mathrm{e}}}{i \omega \mu \phi}}\right|\right)
$$

- Second, when the $S$ - and $T$-modes have reached their dynamic regime in the pores, they still remain at quasi-static regime in the micropores. Hence, the microporous domain is simply described (at the leading order) by its intrinsic permeability $\mathcal{K}_{\mathrm{m}}$ and isothermal compressibility $\frac{\phi}{P^{\mathrm{e}}}$ :

$$
\operatorname{div}\left(V_{\mathrm{m}}\right)+i \omega \phi_{\mathrm{m}} \frac{P_{\mathrm{m}}}{P^{\mathrm{e}}}=0 ; \quad \mu V_{\mathrm{m}}=-\mathcal{K}_{\mathrm{m}} \cdot \nabla P_{\mathrm{m}}
$$


Consequently, the pressure in $\Omega_{\mathrm{sm}}$, results in a field of $P_{2}$ diffusive waves of wavelength $\Lambda_{\mathrm{m}}$ :

$$
\frac{\Lambda_{\mathrm{m}}}{2 \pi}=O\left(\left|\sqrt{\frac{\mathcal{K}_{\mathrm{m}} P^{\mathrm{e}}}{i \omega \mu \phi_{\mathrm{m}}}}\right|\right)
$$

The interesting situation arises when $\frac{\Lambda_{\mathrm{m}}}{2 \pi}$ is of the order of the microporous domain size, $d_{\mathrm{sm}}$. In that case, occurring for frequencies $\omega=O\left(\frac{\mathcal{K}_{\mathrm{m}} P^{\mathrm{e}}}{d_{\mathrm{sm}}^{2} \mu \phi}=\omega_{d}\right)$, the pressure in $\Omega_{\mathrm{sm}}$ is in transient regime. Consequently, the order of the pressure gradient in $\Omega_{\mathrm{sm}}$ is $O\left(\frac{2 \pi P_{\mathrm{m}}}{\Lambda_{\mathrm{m}}}\right)$, while in the media $\nabla^{0} P=O\left(\frac{2 \pi^{0} P}{\Lambda}\right)$. Noticing that the continuity of pressure at the interface $\Gamma$ between the pores and the microporous domain imposes that ${ }^{0} P$ and $P_{\mathrm{m}}$ are of the same order of magnitude, one derives the estimate of the contrast of the velocities in pores and micropores:

$$
\frac{V_{\mathrm{m}}}{{ }^{0} V}=O\left(\frac{\mathcal{K}_{\mathrm{m}} \cdot \nabla P_{\mathrm{m}}}{{ }^{0} K \cdot \nabla^{0} P}\right)=O\left(\sqrt{\frac{\mathcal{K}_{\mathrm{m}} \phi}{{ }^{0} K \phi_{\mathrm{m}}}}\right)=O\left(\sqrt{\frac{\mathcal{K}_{\mathrm{m}}}{\mathcal{K}}}\right)
$$

Consequently, the conditions of emergence of double-porosity effects are reached when:

$$
\mathcal{K}_{\mathrm{m}}=O\left(\epsilon^{2} \mathcal{K}\right), \omega_{v m}=O\left(\epsilon^{2} \omega_{v}\right) ; \text { thus } V_{\mathrm{m}}=\epsilon O\left({ }^{0} V\right), \Lambda_{\mathrm{m}}=O(\epsilon \Lambda)=O\left(d_{\mathrm{sm}}\right)
$$

\subsection{Homogenized Description of Double-Porosity Media}

Since the microporous skeleton is treated as its equivalent Darcy medium, in the second twoscale homogenization, the $y$ variable accounts for the fluctuations of the physical variables within the pores and of the "mean" variables $V_{\mathrm{m}}, P_{\mathrm{m}}$ within the microporous domain.

Consequently, in the pores $\Omega_{\mathrm{f}}$, the physics is still described by the set (5-6-7). The only modification with single-porosity concerns the boundary conditions on the interface $\Gamma$, that expresses now the continuity of flux, pressure and temperature (i.e., isothermal condition since this condition prevails in $\Omega_{\mathrm{sm}}$ ):

$$
v_{/ \Gamma}=V_{m / \Gamma} ; \quad p_{/ \Gamma}=P_{m / \Gamma} ; \quad \theta_{/ \Gamma}=0
$$

In the microporous domain $\Omega_{\mathrm{sm}}$, the physics is governed by (34). However, accounting for the estimates (35), and taking the macroscopic wavelength as reference length, the flux term have to be rescaled by $\epsilon^{2}$ and the differential system reads:

$$
\begin{gathered}
-\epsilon^{2} \operatorname{div}\left(\frac{\mathcal{K}_{\mathrm{m}}}{\mu} \cdot \nabla P_{\mathrm{m}}\right)+i \omega \phi_{\mathrm{m}} \frac{P_{\mathrm{m}}}{P^{\mathrm{e}}}=0 ; \mu V_{\mathrm{m}}=-\epsilon^{2} \mathcal{K}_{\mathrm{m}} \cdot \nabla P_{\mathrm{m}} \\
P_{\mathrm{m}}(x, y)={ }^{0} P_{\mathrm{m}}(x, y)+\epsilon{ }^{1} P_{\mathrm{m}}(x, y)+\cdots ; V_{\mathrm{m}}(x, y)=\epsilon{ }^{1} V_{\mathrm{m}}(x, y)+\epsilon^{2}{ }^{2} V_{\mathrm{m}}(x, y)+\cdots
\end{gathered}
$$

In this frame work, we again proceed to homogenization. In the pores, the fields ${ }^{0} P,{ }^{0} \theta,{ }^{1} p$, ${ }^{0} v$, are unaffected by the microporosity (simply because the balance equations and the boundary conditions are unchanged up to this order). Now, in the microporous domain, the local field ${ }^{0} P_{\mathrm{m}}$, is driven by the set:

$$
S_{\mathrm{m}}^{0}\left\{\begin{array}{c}
-\operatorname{div}_{y}\left(\frac{\mathcal{K}_{\mathrm{m}}}{\mu} \cdot \nabla_{y}{ }^{0} P_{\mathrm{m}}\right)+i \omega \phi_{\mathrm{m}} \frac{{ }^{0} P_{\mathrm{m}}}{P^{\mathrm{e}}}=0 \\
{ }^{0} P_{m / \Gamma}={ }^{0} P ; \quad{ }^{0} P_{\mathrm{m}} \Omega-\text { periodic }
\end{array}\right.
$$


The solution of this linear problem of transient diffusion of pressure, with ${ }^{0} P(x)$ as forcing term on the border $\Gamma$ of $\Omega_{\mathrm{sm}}$, may be expressed in the form:

$$
{ }^{0} P_{\mathrm{m}}(x, y)={ }^{0} P(x)\left[1+{ }^{0} \xi\left(\frac{y}{\Lambda_{\mathrm{m}}}\right)\right]
$$

The pressure distribution $1+{ }^{0} \xi$, solution for ${ }^{0} P(x)=1$, is complex and frequency dependent. At low frequency $\left(\frac{d_{\mathrm{ms}}}{\Lambda_{\mathrm{m}}} \rightarrow 0\right)$, an uniform distribution identical to the pressure in the pores is reached, ${ }^{0} \xi \rightarrow 0$, that means a full coupling between pores and micropores pressures. At high frequency $\left(\frac{d_{\mathrm{ms}}}{\Lambda_{\mathrm{m}}} \rightarrow \infty\right)$, the pressure tends to zero ${ }^{0} \xi \rightarrow-1$ : except on a boundary layer on $\Gamma$ of the order of $\Lambda_{\mathrm{m}}$, the pores and micropores pressures are uncoupled.

Then, the integration of the zero-order mass balance over pore volume and microporous domain provides

$$
\int_{\Omega_{\mathrm{f}}}\left\{\operatorname{div}_{y}\left({ }^{1} v\right)+\operatorname{div}_{x}\left({ }^{o} v\right)+i \omega\left(\frac{{ }^{0} P}{P^{\mathrm{e}}}-\frac{{ }^{0} \theta}{T^{\mathrm{e}}}\right)\right\} \mathrm{d} \Omega+\int_{\Omega_{\mathrm{sm}}}\left\{\operatorname{div}_{y}\left({ }^{1} V_{\mathrm{m}}\right)+i \omega \phi_{\mathrm{m}} \frac{{ }^{0} P_{\mathrm{m}}}{P^{\mathrm{e}}}\right\} \mathrm{d} \Omega=0
$$

Using the divergence theorem, the continuity of ${ }^{1} v$ and ${ }^{1} V_{\mathrm{m}}$ on $\Gamma$, and the periodicity:

$$
\int_{\Omega_{\mathrm{f}}} \operatorname{div}_{y}\left({ }^{1} v\right) \mathrm{d} \Omega+\int_{\Omega_{\mathrm{sm}}} \operatorname{div}_{y}\left({ }^{1} V_{\mathrm{m}}\right) \mathrm{d} \Omega=\int_{\partial \Omega_{\mathrm{f}}}{ }^{1} v \cdot n \mathrm{~d} s+\int_{\partial \Omega_{s m}}{ }^{1} V_{\mathrm{m}} \cdot n \mathrm{~d} s=0
$$

and, reporting the local fields, one obtains the macroscopic description at the leading order:

$$
\begin{gathered}
\operatorname{div}\left({ }^{0} V\right)+i \omega\left[\frac{{ }^{0} P}{P^{\mathrm{e}}}\left(\phi+\phi_{\mathrm{m}}(1-\phi)\left(1+{ }^{0} D\right)\right)-\frac{{ }^{0} T}{T^{\mathrm{e}}}\right]=0 ; \\
\mu^{0} V=-{ }^{0} K . \nabla{ }^{0} P ; \quad \frac{{ }^{0} T}{T^{\mathrm{e}}}={ }^{0} \Pi \frac{{ }^{0} P}{P^{\mathrm{e}}} ; \quad{ }^{0} D=\frac{1}{\Omega_{s m}} \int{ }_{\Omega_{s m}}^{0} \xi \mathrm{d} \Omega
\end{gathered}
$$

where, ${ }^{0} K$ and ${ }^{0} \Pi$ are the effective parameters of the single porosity media (in the absence of microporosity) and the function $1+{ }^{0} D$ expresses the contribution of the phenomena in the microporous domain to the global mass balance.

Both functions ${ }^{0} D$ and ${ }^{0} \Pi$ are issued from a transient-diffusion process (in the complementary domains $\Omega_{\mathrm{sm}}$ and $\Omega_{\mathrm{f}}$ ). Hence, both present similar features: at low frequencies, ${ }^{0} D \rightarrow 0$ (more precisely $\frac{{ }^{0} D}{i \omega} \rightarrow \frac{\phi_{\mathrm{m}}}{P} \frac{\mu}{\mathcal{K}_{\mathrm{m}}} \mathcal{D}_{\mathrm{m}}$, where $\mathcal{D}_{\mathrm{m}}=O\left(d_{\mathrm{sm}}^{2}\right)$ could be designed as a "diffusion permeability") and the media behaves as a single-porosity medium of total porosity $\phi+\phi_{\mathrm{m}}(1-\phi)$; at high frequencies, ${ }^{0} D \rightarrow-1$, and the medium behaves as a single-porosity medium of porosity $\phi$. Low and high frequency domains are delimited by the critical frequency $\omega_{d}$ that differs from $\omega_{v}=O\left(\omega_{t}\right)$ :

$$
\omega_{d}=\frac{\mathcal{K}_{\mathrm{m}}}{\mathcal{D}_{\mathrm{m}}} \frac{P^{\mathrm{e}}}{\mu \phi_{\mathrm{m}}} ; \quad \frac{\omega_{d}}{\omega_{v}}=\frac{\mathcal{K}_{\mathrm{m}} \mathcal{K}}{\mathcal{D}_{\mathrm{m}}} \frac{P^{\mathrm{e}} \rho^{\mathrm{e}} \alpha_{\infty}}{\mu^{2} \phi \phi_{\mathrm{m}}}
$$

\subsection{Comment on Non-locality}

Despite the presence of inner dynamics for the pressure at the REV scale, the zero-order description of the double-porosity medium remains "local in space". Naturally, an enhanced description accounting for scattering would introduce non-local correctors in space.

In fact, similarly to the $S$ - and $T$-modes in the pores, the transient diffusion mode of pressure $\left(P_{2}\right.$-mode) is confined within the microporous domain. Consequently, at the macroscale, this results into an additional parameter non-local in time expressing the frequency 
dependance of the inner dynamics of pressure localized in $\Omega_{\mathrm{sm}}$. The $P_{2}$-mode in the microporous domain introduces a characteristic time $\tau_{d}=O\left(1 / \omega_{d}\right)$ different than those related to $T$ - and $S$-modes in the pores. The physical mechanism may be summarized as follows. The carrier macroscopic wave still induces a quasi-static regime of the $P$-mode within the pores. This implies a uniform pressure in pores that excites the $P_{2}$-mode in the microporous domain, and in turn induces a transient source of flux in the pores.

The interest for sound absorbing materials is that, according to the morphological and mechanical parameters of $\Omega_{\mathrm{sm}}$, this new diffusion mechanism induces an increase of dissipation that may occur at frequency $O\left(\omega_{d}\right)$ significantly lower frequency than the critical frequency of the single porosity medium (Only and Boutin 2003).

\section{Conclusion}

The study by homogenization of wave propagation in rigid air saturated porous media in different situations, enables to clarify how the (non)-locality - in time and/or space-of the macroscopic description is related to the regime of the phenomena that occur at the ERV scale. Both non-locality effects reveal the loss of a perfect quasi-static equilibrium free of volume loading at the local scale, but for different reasons.

It appears that the macro non-locality in time is due to modes in transient regime at the $E R V$ scale, or equivalently having a wavelength of the order of the ERV size. In other words, this effect arises at the leading order when the frequency of the macroscopic carrier wave coincides (in order of magnitude) with the eigen frequency of the locally transient phenomena. At lower frequency, the mode degenerates, the regime remains quasi-static at the ERV scale and the macro description tends to be local in time (or weakly non-local).

The non-locality in time, revealed practically by memory effects, implies that the dynamics deviates from the Newtonian dynamics Auriault (1980), and that the thermal law departs from the classical thermodynamics. This comes from the coexistence of transient regimes at both local and macro scale. Such a situation can also be encountered in elastic composites with strong contrasts of properties as demonstrated in the pioneer paper (Auriault and Bonnet 1985). In that latter case, the deviation from Newtonian dynamics is induced by local resonances and leads to band gap phenomena. By analogy with electromagnetism, this type of material is now referred to as meta-materials. In poroacoustics, the local modes are related to diffusion process, involving viscous deviatoric stress $(S$-mode), and thermal flux ( $T$-mode) for single-porosity media but also pressure diffusion $\left(P_{2}\right.$-mode) in double-porosity media. The diffusive nature of the modes avoids the apparition of inner resonance and the macro waves propagate whatever the frequency. Nevertheless, porous media could be considered as a particular type of meta materials.

Note finally, that a simple manner to obtain transient regimes at both macro and local scale (hence non-locality in time) is to consider several phenomena involving strong contrasts of parameters. This applies to the present case since, for single porosity, $\omega \mu / P^{\mathrm{e}} \ll$ $1, \omega \kappa / c_{p} P^{\mathrm{e}} \ll 1$ and for double porosity $\mathcal{K}_{\mathrm{m}} / \mathcal{K} \ll 1$.

Even if in acoustics, non-locality in space combines with non-locality in time, and if, mathematically, both can be addressed in a similar manner - through convolution product - they are physically of different nature.

Non-locality in space is intrinsically related to the non-homogeneity of the macro fields. The assumption of perfect scale separation, imposes implicitly the homogeneity of the macro fields and gives the leading order behavior, local in space. Non-homogeneous macro-fields 
deviate from this perfect case and the macro description requires to be improved by the effects of higher gradients. These latter modify the local equilibrium, by adding source terms neglected at the leading order. Hence, the reason of the change of equilibrium is due to spatial variation and not (directly) to transient effect.

Conversely to memory effects, that may be of the leading order, only weak non-locality in space has been derived. This relies on the fact, that a non-locality in space at the leading order, would mean a pressure field fluctuating at both local and global scales, which would be in contradiction with the requirement of scale separation. Hence, the homogenization only provides the weak non-local correctors of the local behavior.

Besides, notice that the homogenization method evidences links between, on the one hand, the theories of higher gradient or micromorphic material (Eringen 1968) mainly developed for statics in solid mechanics, and, on the other hand, the description of Rayleigh scattering in heterogeneous media (Ishimaru 1997; Sheng 1995). Further, this study involves phenomena with memory effects not considered in the classical approaches of scattering (Gubernatis 1977; Hirsekorn 1988; Stanke and Kino 1984).

To conclude, the generality and the convergence of the arguments lead to infer that the conclusions drawn on the relations between the time/space non-locality and the regime of the phenomena at the ERV scale, may apply to other physics in heterogeneous media, provided that the scale separation is respected.

\section{References}

Allard, J.-F.: Propagation of sound in porous media. Elsevier, New York (1993)

Attenborough, K.: Acoustical characteristics of rigid fibrous absorbents and granular media. J. Acoust. Soc. Am. 73(3), 785-799 (1983)

Auriault, J.L.: Dynamic behaviour of a porous medium saturated by a Newtonian fluid. Int. J. Engng Sci. 18, 775-785 (1980)

Auriault, J.L.: Heterogeneous medium. Is an equivalent macroscopic description possible. Int. J. Engng Sci. 29(7), 785-795 (1991)

Auriault, J.L., Bonnet, G.: Dynamique des composites élastiques périodiques. Arch. Mech. 37(4-5), 269-284 (1985)

Auriault, J.L., Boutin, C.: Deformable porous media with double porosity. III: Acoustics. Transp. Porous Media 14, 143-162 (1994)

Auriault, J.L., Geindreau, C., Boutin, C.: Filtration law in porous media with poor scale separation. Transp. Porous Media. 60(1), 89-108 (2005)

Auriault, J.-L., Boutin, C., Geindreau, C.: Homogenization of coupled phenomena in heterogenous media. ISTE and Wiley, London (2009)

Barenblatt, G.I., ZheltovI, P.: On fundamental equations of flow of homogeneous liquids in naturally fractured rocks. Dokl. Akad. Nauk. USSR 132(3), 545-548 (1960)

Benssoussan, A. Lions J.L., Papanicolaou, G.: Asymptotic methods in periodic structures. North-Holland, Amsterdam (1978)

Biot, M.A. (1956) Theory of propagation of elastic waves in a fluid-saturated porous solid. I. Low-frequency range, II. Higher Frequency, J. Acoust. Soc. Am 28, 168-178;179-191

Boutin, C.: Microstructural influence on heat conduction. Int. J. Heat Mass Transf. 38(17), 3181-3195 (1995)

Boutin, C.: Microstructural effect in elastic composite. Int. J. Solids Struct. 33(7), 1023-1051 (1996)

Boutin, C.: Rayleigh scattering of acoustic wave in rigid porous media. J. Am. Soc. Acoust. 122(4), 1888-1905 (2007)

Boutin, C., Auriault, J.L.: Dynamic behaviour of porous media saturated by a viscoelastic fluid. Application to bituminous concrete. Int. J. Engng. Sci. 28(11), 1157-1181 (1990)

Boutin, C., Auriault, J.L.: Rayleigh scattering in elastic composite materials. Int. J. Eng. Sci. 31(12), 16691689 (1993)

Boutin, C., Royer, P., Auriault, J.L.: Acoustic absorption of porous surfacing with dual porosity. Int. J. of Solids Struct. (Special Issue on Poroelasticity) 35(34-35), 4709-4737 (1998) 
Champoux, Y., Allard, J.F. Dynamic tortuosity and bulk modulus in air saturated porous media. J. Appl. Phys. 70, 1975-1979 (1991)

Eringen, A.C.: Mechanics of micromorphic continua. In: Kröner, E. (ed.) IUTAM Symposium, pp. 18-35. Springer, Berlin (1968)

Gubernatis, J.E., Domany, E., Krumhansl, J.A., Hubernam, M.: The Born approximation in the theory of the scattering of elastc waves by flaws. J. Appl. Phys. 48, 2812-2819 (1977)

Hirsekorn, S. (1988) The scattering of ultrasonic waves by multiphase polycrystals. J. Acoust. Soc. Am. 83(4), $1231-1242$

Ishimaru, A.: Wave propagation and scattering in random media. IEEE/Oxford University press Classic Reissue, Piscataway (1997)

Leclaire, P., Kelders, L., Lauriks, W., Glorieux, C., Thoen, J.: Determination of viscous characteristics length in air filled porous material by ultrasonic attenuation. J. Am. Soc. Acoust. 99(4), 1944-1948 (1996)

Only, X., Boutin, C.: Acoustic wave propagation in double porosity media. J. Am. Soc. Acoust. 113(6), 73-89 (2003)

Sanchez Palencia, E. Nonhomogeneous media and vibration theory. Lectures notes in Physics, 127, Springer, Berlin (1980)

Sheng, P.: Introduction to wave scattering. Localization and mesoscopic phenomena. Academic press, San Diego (1995)

Stanke, F., Kino, G.: A unified theory for elastic wave propagation in polycristalline materials. J. Acoust. Soc. Am. 75(3), 665-681 (1984)

Tournat, V. Pagneux, V. Lafarge, D., Jaouen, L.: Multiple scattering of acoustic waves and porous absorbing media. Phys. Rev. E. 70(026609), 1-9 (2004)

Warren, J.R., Root, P.J.: The behavior of naturally fractured reservoirs. Soc. Pet. Eng. J. 3(3), 245-255 (1963)

Zwikker, C., Kosten, W.: Sound absorbing materials. Elsevier, Amsterdam (1949) 